\title{
PwRn I, a novel Ty3/gypsy-like retrotransposon of Paragonimus westermani: molecular characters and its differentially preserved mobile potential according to host chromosomal polyploidy Young-An Bae ${ }^{1}$, Jong-Sook Ahn², Seon-Hee Kim', Mun-Gan Rhyu' ${ }^{2}$, Yoon Kong ${ }^{1}$ and Seung-Yull Cho*1
}

Address: ${ }^{1}$ Department of Molecular Parasitology and Samsung Biomedical Research Institute, Sungkyunkwan University School of Medicine, Suwon, Gyeonggi-do 440-746, Korea and 2Department of Microbiology, College of Medicine, Catholic University of Korea, Seoul 137-701, Korea

Email: Young-An Bae - yabae@med.skku.ac.kr; Jong-Sook Ahn - jsa31@cam.ac.uk; Seon-Hee Kim - sunny14@med.skku.ac.kr; MunGan Rhyu - rhyumung@cmc.cuk.ac.kr; Yoon Kong - ykong@med.skku.ac.kr; Seung-Yull Cho* - seungyl@med.skku.ac.kr

* Corresponding author

Published: 14 October 2008

BMC Genomics 2008, 9:482 doi:10.1 186/1471-2164-9-482
Received: 14 May 2008

Accepted: 14 October 2008

This article is available from: http://www.biomedcentral.com/I47I-2I64/9/482

(c) 2008 Bae et al; licensee BioMed Central Ltd.

This is an Open Access article distributed under the terms of the Creative Commons Attribution License (http://creativecommons.org/licenses/by/2.0), which permits unrestricted use, distribution, and reproduction in any medium, provided the original work is properly cited.

\begin{abstract}
Background: Retrotransposons have been known to involve in the remodeling and evolution of host genome. These reverse transcribing elements, which show a complex evolutionary pathway with diverse intermediate forms, have been comprehensively analyzed from a wide range of host genomes, while the information remains limited to only a few species in the phylum Platyhelminthes.
\end{abstract}

Results: A LTR retrotransposon and its homologs with a strong phylogenetic affinity toward CsRn I of Clonorchis sinensis were isolated from a trematode parasite Paragonimus westermani via a degenerate PCR method and from an insect species Anopheles gambiae by in silico analysis of the whole mosquito genome, respectively. These elements, designated PwRnI and AgCR-I - AgCR-I4 conserved unique features including a t-RNATrp primer binding site and the unusual CHCC signature of Gag proteins. Their flanking LTRs displayed $>97 \%$ nucleotide identities and thus, these elements were likely to have expanded recently in the trematode and insect genomes. They evolved heterogeneous expression strategies: a single fused ORF, two separate ORFs with an identical reading frame and two ORFs overlapped by -I frameshifting. Phylogenetic analyses suggested that the elements with the separate ORFs had evolved from an ancestral form(s) with the overlapped ORFs. The mobile potential of $P w R n I$ was likely to be maintained differentially in association with the karyotype of host genomes, as was examined by the presence/absence of intergenomic polymorphism and mRNA transcripts.

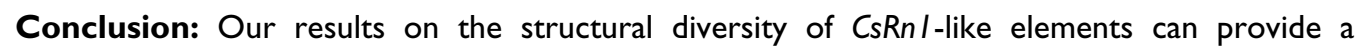
molecular tool to dissect a more detailed evolutionary episode of LTR retrotransposons. The $P w R n I$-associated genomic polymorphism, which is substantial in diploids, will also be informative in addressing genomic diversification following inter-/intra-specific hybridization in P. westermani populations. 


\section{Background}

Retrotransposons, which comprise a major portion of eukaryotic genomes, replicate progeny copies into new genomic loci via reverse transcription of an RNA intermediate [1]. As intragenomic parasites, retrotransposons have been known as a potent causative agent involved in various harmful biological processes such as insertional mutagenesis [2] and ectopic recombination [3]. Conversely, cumulative data have evidenced that these elements play significant roles in the formation and maintenance of host chromosomes [4]. The sporadic expansion of retrotransposons can also introduce genomic/phenotypic variants, which lead to speciation in the long evolutionary terms [5,6]. Retrotransposons are subdivided into two large categories, long-terminal-repeat (LTR) and non-LTR retrotransposons, according to overall structures, and the elements with LTR seem to be the most abundant type in invertebrates. The LTR retrotransposons include multiple groups, as have been distinguished by comparing their own gene products $[7,8]$.

In addition to the typical LTR retrotransposons, multiple elements with unusual structural features have recently been isolated from various genomes, such as CsRn1 of Clonorchis sinensis [9], Xena of Takifugu rubripes [10], Gmr1 of Gadus morhua [11] and Saci-2 of Schistosoma mansoni [12]. The CsRn1 and Saci-2 elements were found to encode Gag with unique motifs of $\mathrm{CHCC}$ and $\mathrm{CCCH}$, respectively, instead of the conventional CCHC. Gmr1 produced a Pol protein, in which the functional protein domains lie in an order identical to those of Ty1/copia elements (protease [PR]-integrase [IN]-reverse transcriptase [RT]-RNase $\mathrm{H}[\mathrm{RH}])$. Retrotransposons of the most ancient Xena group had a single open reading frame (ORF) containing a RT domain but lacking any other enzymatic domain. Taken together, these facts have suggested that the category and evolutionary episode of the diverse reverse-transcribing elements are more complex than currently understood.

Paragonimus westermani is a hermaphroditic, digenetic trematode that lives as adult in the lungs of carnivorous mammals. This parasite causes pulmonary and cerebral diseases in humans and is one of the most medically important flukes. The natural populations of P. westermani in northeast Asian countries have three different levels of polyploidy in their genomic contents, i.e., di-, tri- and tetrapolyploidy, displaying variations in morphology, allozyme patterns, and nucleotide sequences of ribosomal and mitochondrial DNAs [13]. The Paragonimus genome contains retrotransposons, which belong to various retrotransposon groups including the CsRn1 clade [14]. CsRn1like retrotransposons have been detected largely in trematodes and insects $[9,12,15]$. With the unique Gag motif and amino acid conservation, members of the clade showed heterogeneity in their coding profiles. Furthermore, Boudicca and Saci-3 of S. mansoni contain a third ORF resembling envelope protein (ENV) of errantivirus and retrovirus $[12,16]$.

The genomic distribution of CsRn1-like elements have been well described in $C$. sinensis and $S$. mansoni, with their unique structural features $[9,12,16]$. Nevertheless, molecular information on this distinctive clade is highly limited to address their evolutionary episodes. In this study, we isolated a novel CsRn1-like LTR retrotransposon in the P. westermani genome and analyzed its intra- and inter-genomic distribution patterns among the parasite populations with different karyotes. Differential replication activity of the Paragonimus element was examined by detecting the presence or absence of mRNA transcripts and intergenomic polymorphism introduced by the element. The molecular characters of multiple retrotransposons homologous to the element were also analyzed from the genomes of an African malaria mosquito Anopheles gambiae, which had recently been released [17], and a fruit fly Drosophila melanogaster, in order to gain more detailed information on the invertebrate-specific CsR $n 1$ clade. The heterogeneous expression strategies were found to be substantial within the unique clade, and the possible evolutionary relationships between elements with distinctive coding profiles were shown by phylogenetic analyses.

\section{Results \\ Isolation of CsRn I-like retrotransposons from $\mathbf{P}$. westermani and A. gambiae}

Using the previously described degenerate primers, retrotransposon-related sequences had been isolated in $P$. westermani [14]. These sequences represented various LTR retrotransposons belonging to the multiple clades of Metavirus (Ty3/gypsy group) including the CsRn1 clade, of which members seemed to expand uniquely in lower animal taxa such as Insecta and Trematoda $[9,12]$. One clone (Pw-D-13 [GenBank:BZ715464]) showing the highest sequence identity with $C s R n 1$ was used as a probe in the screening of the genomic DNA library of the parasite. As shown in Figure 1, the full-unit LTR retrotransposon encompassing the probe sequence was determined and named PwRn1 ( $\underline{P}$. westermani Retrotransposon 1 ) by comparing sequences of two positive clones ( $\lambda \mathrm{Pw}-13-1$ [GenBank:AY237161] and $\lambda$ Pw-13-2 [GenBank:AY237162]). The PwRn1 copy in $\lambda$ Pw-13-2 was 5,400-bp long and was bound by direct repeats of 4 bp (5'-GGCG-3') known as target site duplication (TSD). The internal sequence was corrupted by several stop codons introduced by indels and/or base substitutions, although its flanking 5'- and 3'LTRs had an identical size (381 bp) and a high degree of sequence identity (99.7\%). The internal coding regions were further retrieved from different PwRn1 copies (35 copies [GenBank:EU622539 - EU622573], Avg. diver- 


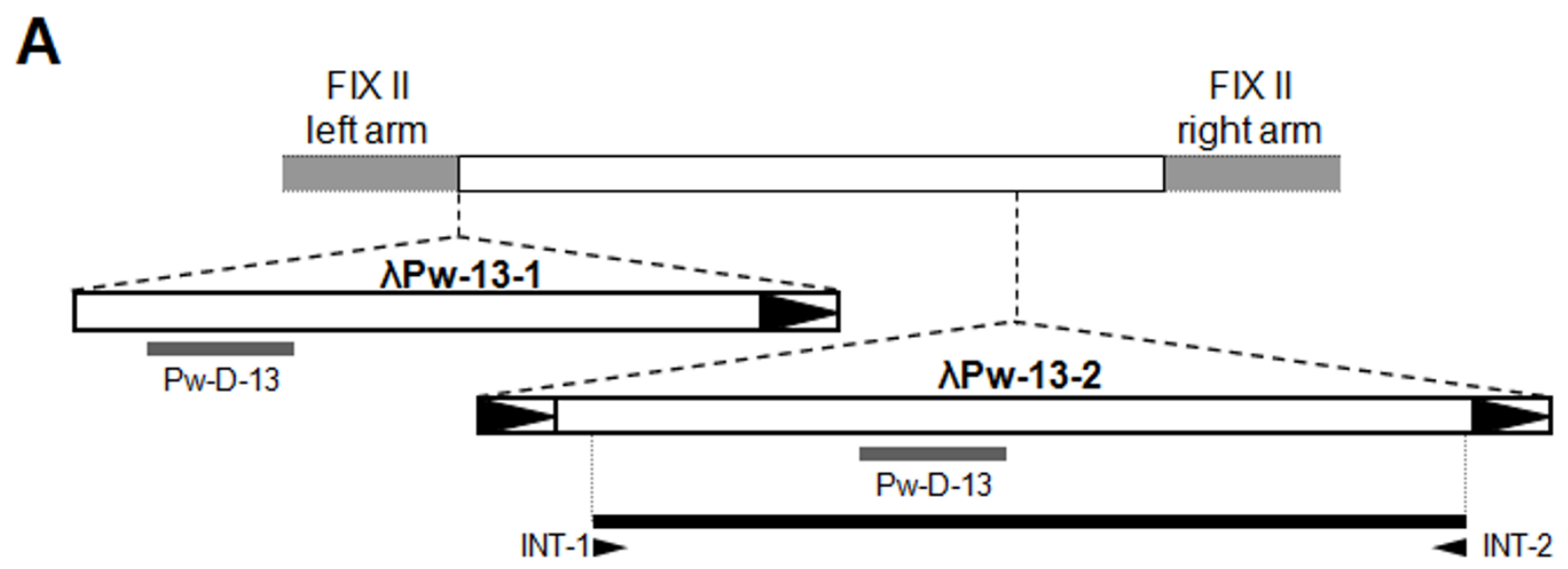

B

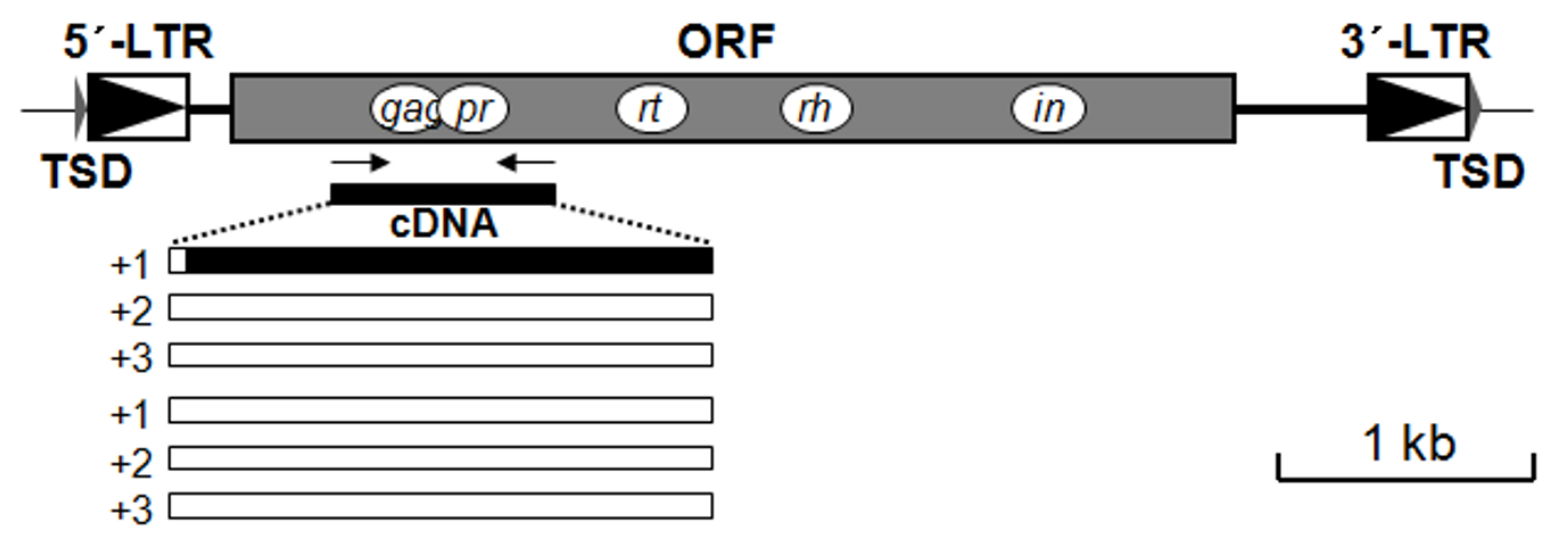

Figure I

Isolation and structural characterization of a CsRn I-like element from the genome of Paragonimus westermani. (A) Schematic representation of an LTR retrotransposon contained in the genomic lambda clones $(\lambda P w-I 3-I$ and $\lambda P w-I 3-2)$, which were homologous to the Pw-D-I 3 probe. Boxes with a filled arrowhead indicate a direct repeat sequence. The primer positions used in the retrieval of multiple copy sequences are also shown (INT-I, 5'-AGTTGGAAGCCACAT-

TCGCGCTACG-3' and INT-2, 5'-GTGACAACAACCCTTCGATCCTGATG-3'). (B) Overall structure of the Paragonimus retrotransposon, named PwRn I. Gray box represents an open reading frame (ORF) encoding Gag, protease (PR), reverse transcriptase (RT), RNase $\mathrm{H}(\mathrm{RH})$ and integrase (IN). Duplicated target sites of 4 bp are indicated as target site duplication (TSD). The gag-pol boundary region was further verified by the nucleotide sequences of cDNA clones (see also the legend for Figure 6).

gence $=0.051 \pm 0.002)$ (Figure $1 \mathrm{~A})$. The coding profile of PwRn1 was predicted with one of these sequences (PwRn1-Int-29 [GenBank:EU622567]), which contained the longest ORF of 3,585 bp encoding a single 1194-aa polypeptide. The corrupted nucleotides in the 5'- and 3'upstream regions of the ORF were corrected by comparing them with the equivalent regions in a consensus sequence determined from the 35 sequences. The gag-pol boundary region was further confirmed by sequencing the corresponding cDNAs (Figure 1B).
The genomic sequences of A. gambiae in the GenBank database were examined by using the amino acid sequence of CsRn1 Gag protein as a query (TBLASTN algorithm). The nucleotide sequences of matching entries were analyzed via a series of BLASTN searches to determine the full-unit retrotransposons encompassing each of the gag sequences. The integrity and identity of the elements were verified by detecting a TSD pair at the boundary regions, and by comparing lengths and nucleotide sequences of their LTRs, respectively. These procedures

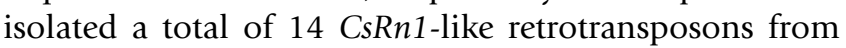
the mosquito genome, which were designated AgCR-1 - 
AgCR-14 (ㅁ․ gambiae $\underline{C} s R n 1$-like Retrotransposon) (Table $1)$. The complete sequences were used as a BLAST query to retrieve additional copy sequences from the genome. The overall structures and coding profiles were predicted, as described above (Figure 2). One-half of the AgCR elements were found to be identical to the CsRn1-clade members previously reported [15] (Table 1). CsRn1-like elements could not be retrieved from the genomic sequences of $D$. melanogaster, other than the retrotransposon on AE003787 [9], named DmCR-1 in this study.

\section{Functional motifs and structural features of PwRn I and AgCRs}

TSD detected in all of the CsRn1-like elements were found to be $4 \mathrm{bp}$, although there seemed to be no specific or preferential nucleotide in selecting the insertional targets among copies of each element and among the AgCR types (Table 1). The short inverted repeats of $3 \mathrm{bp}$, found at both ends of LTR in most retrotransposons (5'-TGT...ACA3') [18], were slightly modified either to $5^{\prime}$-TGT...AAA-3' or to 5'-TGT...ATA-3' (Additional file 1). The LTR pairs flanking each of the $A g C R$ elements displayed $>97 \%$ nucleotide identities, suggestive of their recent expansion in the mosquito genome [19]. The putative primer-binding site of these elements for the synthesis of the first cDNA strand (5'-TGGTGAG/CCCCGT/A-3') was complementary to the nucleotides at the 3'-end of bovine t-

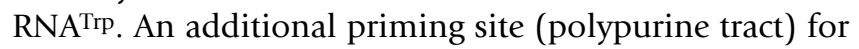
the synthesis of the second cDNA strand was also found in the direct upstream region of 3'-LTR.
The internal regions of these elements contained ORFs with different expression strategies for their autonomous retrointegration. PwRn1 had an ORF similar to that of CsRn1, in which the gag and pol genes were fused together in a single frame (Figure 1). Of the $14 \mathrm{AgCR}$ elements analyzed, nine elements utilized two ORFs overlapped by -1 frameshifting to encode the Gag and Pol proteins, while the other six elements contained two ORFs with an identical reading frame, which were intervened by a short nucleotide sequence ranging from 60 to $165 \mathrm{bp}$ (Figure 2 ). The amino acid sequences of Gag proteins in retrotransposons generally diverge very rapidly so that they display a low level of sequence identity to one another, except for the functional Cys-His signature $\left(\mathrm{CX}_{2} \mathrm{CX}_{4} \mathrm{HX}_{4} \mathrm{C}\right.$; CCHC) [20]. The proteins, however, showed a high degree of amino acid conservation over their entire lengths in these CsRn1-like retrotransposons. They had the unique CHCC signature $\left(\mathrm{CX}_{2} \mathrm{HX}_{9} \mathrm{CX}_{3} \mathrm{C}\right)$ in the $\mathrm{C}$-terminal regions, while it was imperfect in 3 elements, $A g C R-5,-7$ and -9 . The $\alpha$-helix-rich secondary structures of the Gag proteins were similar to those of the other Ty3/gypsy-like LTR retrotransposons (Additional file 2 ). The enzymatic domains contained in the Pol proteins were also well conserved with the conventional motifs/ signatures: DT/SG tripeptide of PR, F/YXDD tetrapeptide of RT, DAS tripeptide of RH, and HHCC and DDE signatures of IN (data not shown). A series of LTR retrotransposons have an additional motif in the $\mathrm{C}$-terminal region of IN, which is implicated in non-specific binding to the target site and/or to LTR via the best-conserved GPY/F residues $[21,22]$. This motif could also be detected in the cor-

Table I: CsRn I-like retrotransposons of Anopheles gambiae isolated in this study

\begin{tabular}{|c|c|c|c|c|c|c|c|c|}
\hline \multirow[t]{2}{*}{ Element } & \multirow[t]{2}{*}{ Contig No. (nucleotide position)a } & \multicolumn{4}{|c|}{ Nucleotide lengths (bp) } & \multirow[t]{2}{*}{ LTR identity (\%) } & \multirow[t]{2}{*}{ TSD } & \multirow[t]{2}{*}{ Note $^{d}$} \\
\hline & & 5'-/3'-LTR & Full unit & ORFIb & ORF2 $2^{b, c}$ & & & \\
\hline$A g C R I$ & AAAB0I008980 (3956962-396I632) & $264 / 264$ & 4671 & 978 & $2961(0)$ & 98.1 & AAGC & \\
\hline$A g C R 2$ & AAAB0I0088I0 (4III3-470I9) & $368 / 368$ & 5907 & 978 & $3168(0)$ & 100 & $\mathrm{AACT}$ & GYPSY5IAG \\
\hline$A g C R 3$ & AAAB0I008968 (I I35463-II39639) & $146 / 146$ & 4177 & 756 & $3090(-1)$ & 100 & AATG & GYPSY50AG \\
\hline AgCR4 & AAAB0I008984 (943696I-9442636) & $356 / 356$ & 5676 & 948 & $3135(0)$ & 100 & AACT & GYPSY70AG \\
\hline AgCR5 & AAAB0I00883I (1206-6507) & $202 / 202$ & 5302 & 882 & $2993(-1)$ & 100 & CGTC & \\
\hline AgCR6 & AAABOI 008880 (20632-25306) & $300 / 300$ & 4675 & 888 & $3087(0)$ & 99.7 & CACC & GYPSY53AG \\
\hline AgCR7 & AAABOI008850 (51014-56635) & $266 / 267$ & 5622 & 876 & $2856(-I)$ & 97.3 & AAGT & \\
\hline AgCR8 & AAAB0I008888 (323। I I 8-3237246) & $394 / 394$ & 6126 & 984 & $3249(0)$ & 100 & GCAT & GYPSY52AG \\
\hline AgCR9 & AAAB0I00885I (1315535-1320623) & $260 / 260$ & 5089 & 915 & $2964(-1)$ & 100 & GGTT & \\
\hline$A g C R / O$ & AAAB0I 008869 (24283-304I8) & $319 / 316$ & 6136 & 885 & $2991(-1)$ & 99.1 & ATGT & GYPSY49AG \\
\hline$A g C R / I$ & AAABOI008957 (186235-192486) & $407 / 407$ & 6252 & 909 & $308 I(-I)$ & 98.5 & TATC & \\
\hline$A g C R / 2$ & AAAB0I008964 (27|4632-2720796) & $370 / 370$ & 6165 & 894 & $3084(-1)$ & 100 & AGTT & GYPSY48AG \\
\hline$A g C R / 3$ & AAAB0I008849 (1694453-1699416) & $219 / 206 \mathrm{e}$ & 4964 & 933 & $2946(-1)$ & 99.5 & AGGGe & \\
\hline$A g C R / 4$ & AAAB0I008944 (738407-744580) & $383 / 383$ & 6174 & 984 & $2970(-1)$ & 100 & $\mathrm{ACCC}$ & \\
\hline
\end{tabular}

aContig sequence containing a representative copy is listed.

bThe lengths of ORFs were estimated from each of the elements, after correcting the corrupted nucleotide sequences (see text).

cNumber in parentheses indicates a shift in the reading frame of ORF2 compared to that of ORFI.

dSynonymous names, as have been designated in a previous report [15].

eNucleotides comprising the end of the 3 '-LTR and TSD were deleted in the contig and thus, TSD sequence found at the 5 '-flanking region was presented. 


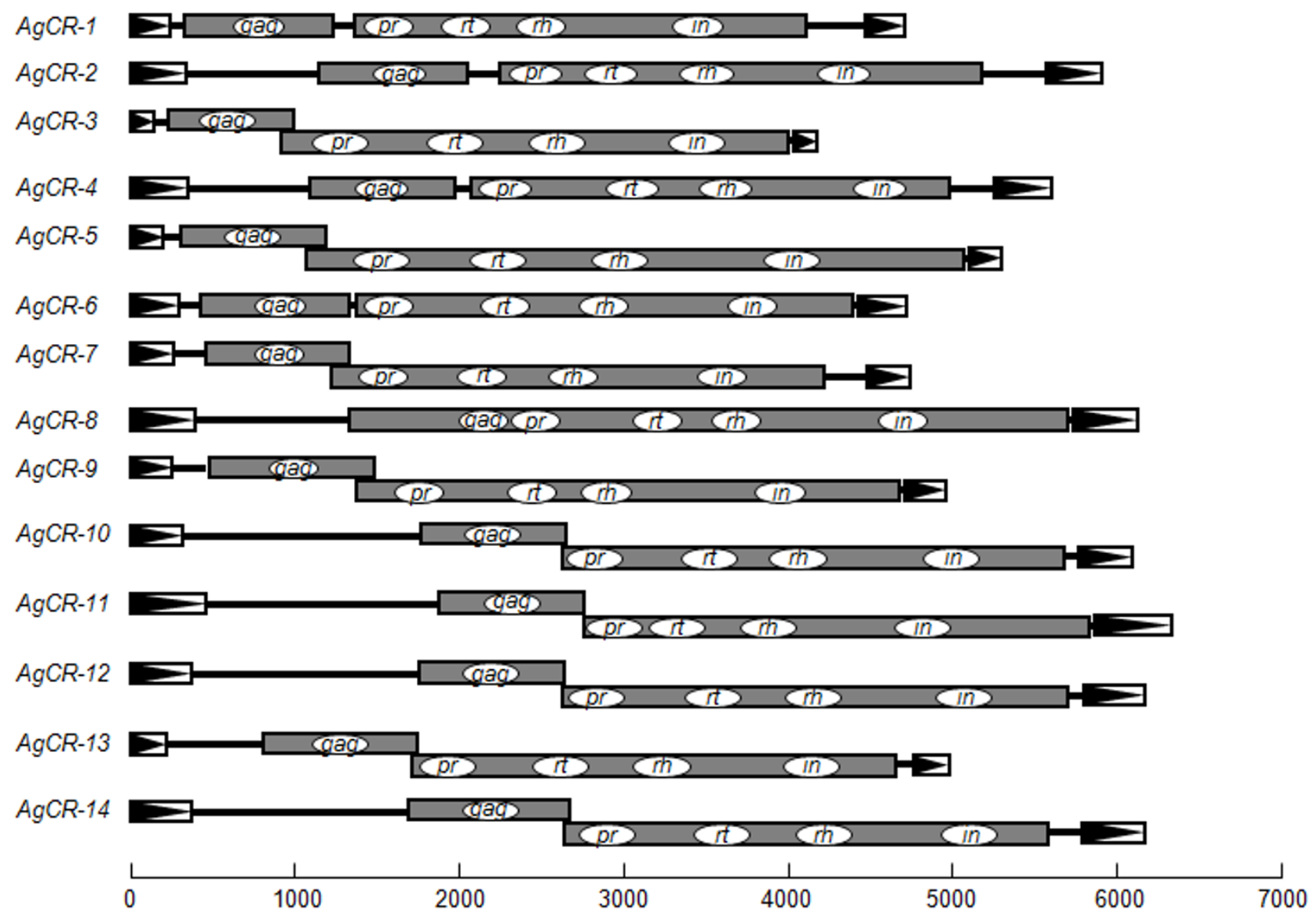

Figure 2

Schematic diagram showing the overall structures and expression strategies of AgCR elements isolated from the genomic sequences of Anopheles gambiae. The coding profiles within each of the elements were predicted from a representative copy, as listed in Table I, of which disrupted bases had been corrected by referencing a consensus sequence of multiple copies.

responding proteins of PwRn1 and AgCRs with the exceptions of $A g C R-7$ and -14 . Neither the chromatin organization modifier domain (chromodomain) nor ENV-like protein was detected in these elements.

\section{Phylogenetic analysis of CsRn I-like retrotransposons}

We obtained an alignment of Pol proteins from a total of 48 elements including the 15 elements isolated in this study. Amino acid positions for the three functional domains (RT, RH and IN; 510 aa) were concatenated and used in a phylogenetic analysis with the quartet-puzzling maximum likelihood method. The representative elements belonging to the previous eight clades of Ty3/gypsylike LTR retrotransposons [22], as well as those of CsRn1 clade $[9,12]$, were selected for the analysis. As shown in Figure 3, each of the sequences was well separated into the corresponding clades with significant quartet puzzling frequencies. The PwRn1 and AgCRs were categorized into the tightly conserved CsRn1 clade with a high level of quartet puzzling support $(91 \%)$, as was predicted by the similarities in the primary structures of the Gag and Pol proteins. $P w R n 1$ formed a subclade with its trematode neighbors (supporting value, 80\%). The AgCR elements were further segregated into four distinct clusters and those with separated ORFs were monophyletically distributed in the tree, even though the supporting value was relatively low (53\%; the elements were marked with $\dagger$ ). Saci-2 of S. mansoni, which encodes an unusual Gag protein with the CCCH motif [12], occupied a unique phylogenetic position. Another tree based on the Gag sequences of CsRn1like elements showed a topology similar to that of the Pol tree (boxed tree in Figure 3), while the phylogenetic relationships especially among the Anopheles elements with different expression strategies could be resolved more evidently with the rapidly evolving Gag sequences. The tree proposed that Anopheles elements with the separated ORFs 


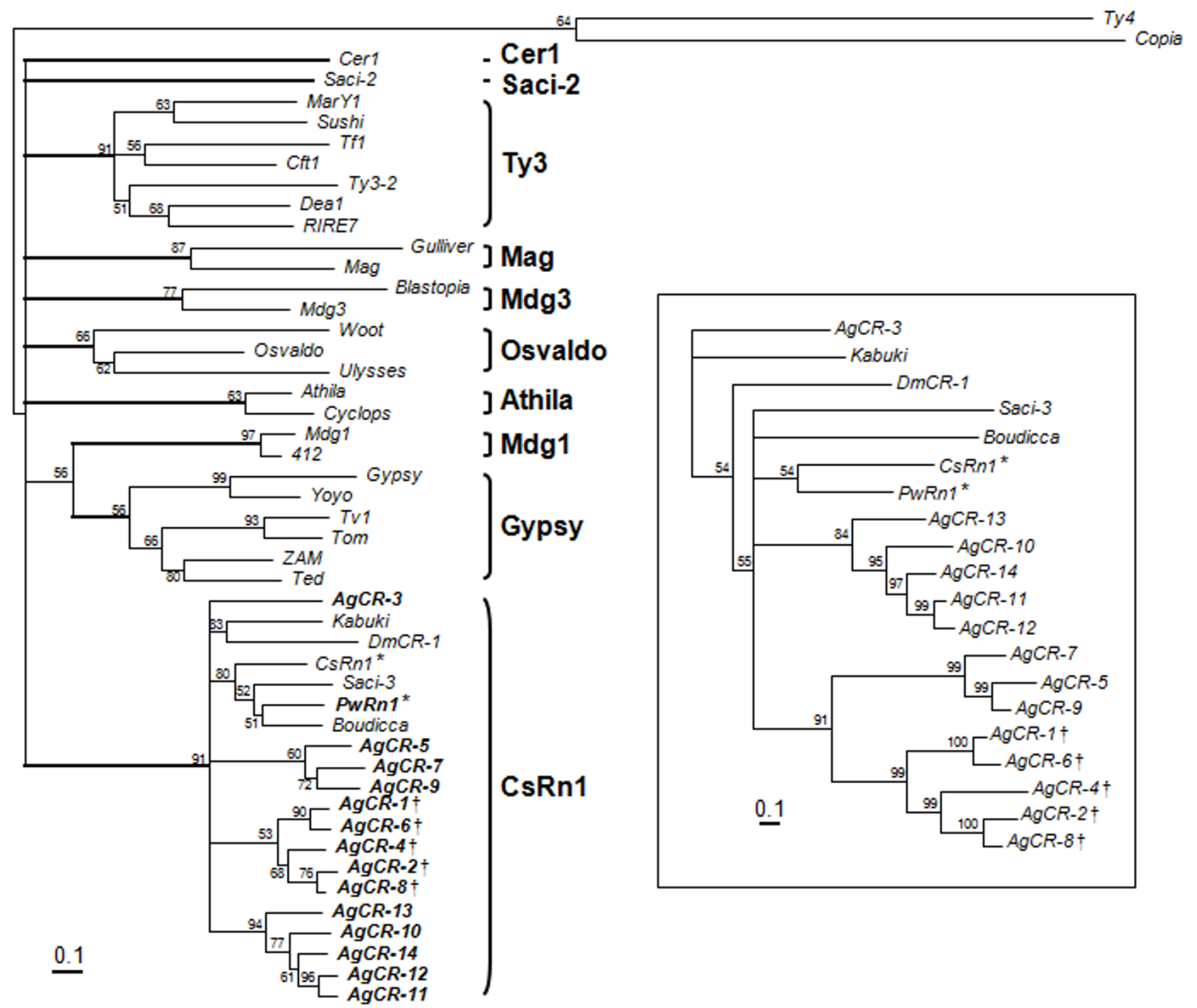

Figure 3

Phylogenetic relationships between the CsRnI-clade members and the other Ty3/gypsy-like retrotransposons. The analysis was performed with a concatenate of reverse transcriptase, RNase $\mathrm{H}$, and $\mathrm{Zn}$ finger and DDE domains of integrase, using the maximum likelihood method of TREE_PUZZLE. The tree was rooted with Ty4 and Copia. Quartet supporting values are presented at each of the branching points. Elements characterized in this study are distinguished by the boldface letters. The symbols $(*, \dagger)$ found at the ends of element names indicate those with a single and two separated open reading frames, respectively. The tree in box was similarly obtained with the Gag sequences of CsRn I-like elements.

have evolved from an ancient form(s) with two overlapped ORFs ( $91 \%$ support).

\section{Genomic distribution and mobile potential of PwRn I}

The copy number of $P w R n 1$ was estimated over 1,000 per haploid Paragonimus genome via dot blotting (Figure 4A). A Southern blot analysis showed that these PwRn1 copies are interspersed throughout the genome, rather than being tandemly arrayed or being clustered at limited loci
(Figure 4B). However, the blotting could not distinguish the distribution patterns of PwRn1 between diploid and triploid genomes, mainly due to the high copy number and resulting smeared hybridization signals. For a more detailed comparison of PwRn1-occupied loci, IRAP analyses were conducted with the individual genomic DNAs and PwRn1 LTR-specific primers (Figure 5). Numerous bands were amplified from genomic loci intervened between two copies of PwRn1. The patterns were found to 




\section{Figure 4}

Copy number and genomic distribution of PwRnI. (A) Reverse dot-blot analysis. The membrane was dotted in duplicate with varying amounts of Pw-D-13 fragment as shown on the top and probed with the genomic DNA of diploids. The blots of cysteine protease (Cys Prot) were used as a single copy control. The signal intensities were compared to estimate the copy number of PwRnI. (B) Southern blotting of PwRnI with the genomic DNAs of diploid (Haenam, $\mathrm{Hn}$ ) and triploid (Bogil-do, Bg) Paragonimus westermani. Restriction endonucleases used for the digestion of DNAs are presented at the top. The positions of DNA size standards (in kb) are shown on the left.

be homogeneous among the triploid individuals and almost all of the triploid markers were detected in at least one of the diploid genomes, especially with the Korean origin (see the bands marked with white circles in Figure 5). Conversely, polymorphic markers were substantially observed among the diploid genomes at intra- and interpopulation levels. Analyses of more parasite individuals (up to 15) showed similar results (data not shown). Given the fact that the sporadic replication of an active retro- transposon introduces intergenomic polymorphism [23], this observation may reflect a differentially preserved mobile potential of PwRn1 depending on the karyotype of its host genome; the element has maintained the ability to produce progeny copies in diploids, whereas it has ceased to retrointegrate into new genomic loci in the triploid populations. In accordance with this suggestion, mRNA transcripts of the element were detected in a diploid population, but not in a triploid one (Figure 6). The intra- 

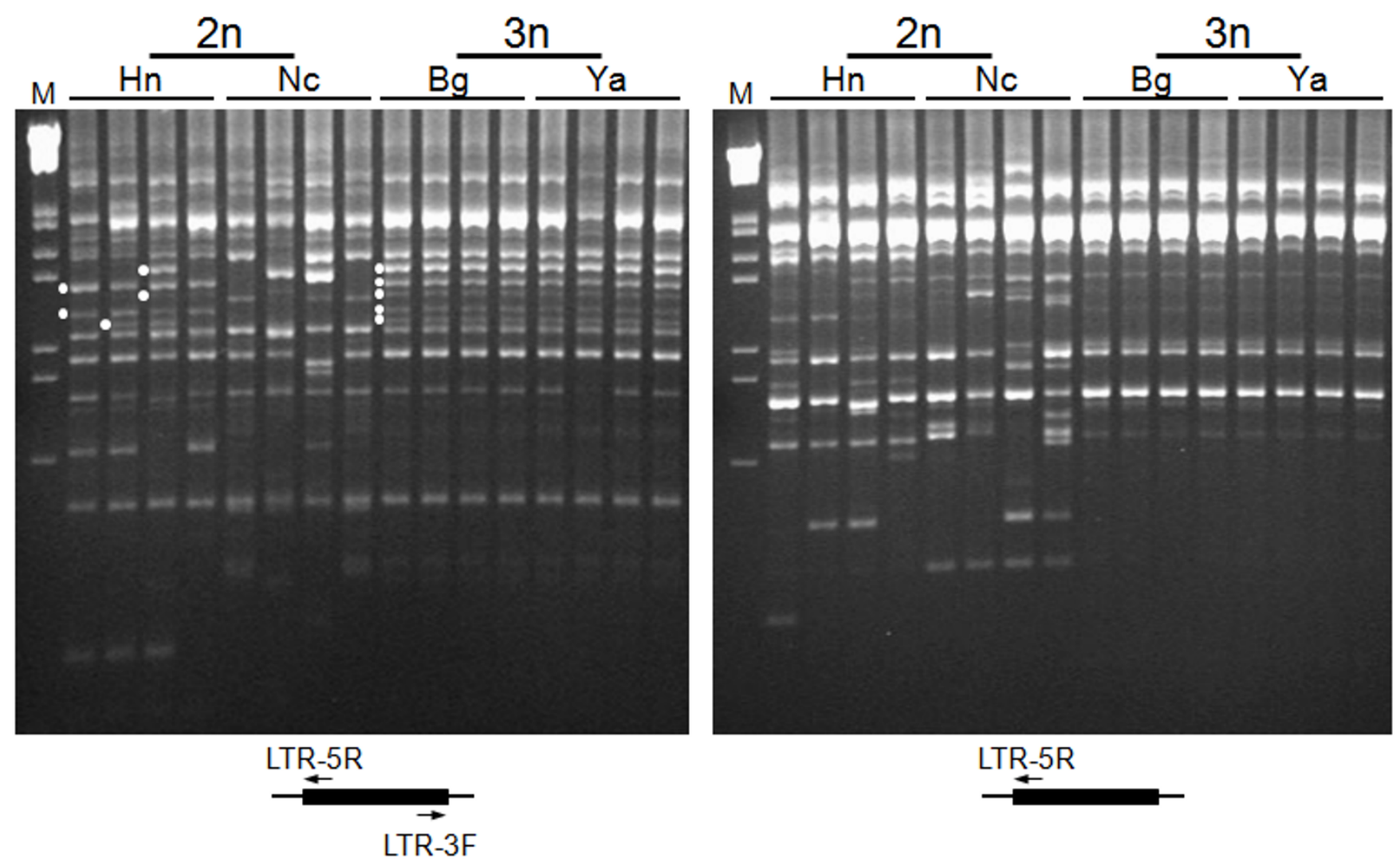

Figure 5

Banding patterns of inter-retrotransposon amplified polymorphism (IRAP). Genomic DNAs were separately extracted from individuals of $P$. westermani (diploids from Haenam, $\mathrm{Hn}$ and Nanchang, $\mathrm{Nc}$; triploids from Bogil-do, Bg and Youngam, Ya). The DNAs were used in PCR with the PwRn I LTR-specific primers, as shown at the bottom. The amplified fragments were electrophoresed on agarose gels and visualized by ethidium bromide staining. White circles indicate triploid genome-specific IRAP markers, which were shared with the Korean diploids. M, lambda DNA/EcoR I + Hind III marker.

genomic expansion of retrotransposons initiates by transcribing their mRNAs using host RNA polymerase. By adapting to this unique replication mode, host defense mechanisms have diversely evolved by focusing on the protection of transcription and/or destruction of mRNA transcripts, including chromatin modification and RNA interference. Therefore, $P w R n 1$ was likely to be suppressed at the initial transcription stage in the triploid population, although no relevant mechanism could be addressed in this study.

\section{Discussion}

Since the first detection of LTR retrotransposons with an unusual capsid protein in a trematode and insect species $[9,24]$, a series of similar elements have been isolated from genomes of the other trematodes and insects. They share unique properties such as PBS for t-RNA ${ }^{\text {Trp }, ~} 4$ bp of TSD and tight amino acid conservation in the encoded proteins. The most conspicuous feature, nevertheless, can be found in the amino acid sequences of Gag proteins. In general, the first ORF contained in various retrotransposons diverge rapidly so that they display low levels of sequence identity to one another, except for the active Cys-His motif $\left(\mathrm{CX}_{2} \mathrm{CX}_{4} \mathrm{HX}_{4} \mathrm{C}\right)$ [20]. However, the proteins of CsRn1-like elements have been known to conserve a novel sequence motif of $\mathrm{CX}_{2} \mathrm{HX}_{9} \mathrm{CX}_{3} \mathrm{C}$ (CHCC), instead of the conventional $\mathrm{CCHC}$ motif. These facts have suggested that members of this clade are originated from an intermediate form of reverse-transcribing elements, which have emerged in the common ancestor of the monophylyl ecdysozoan and lophotrochozoan animals. Retrotransposons characterized from the lung fluke and mosquito possessed all of these features and thus, further supported the notion that these elements are selectively expanded in the lower animal taxa.

The Gag protein of retrotransposons functions as a nucleocapsid protein during the assembly of virus-like particle 


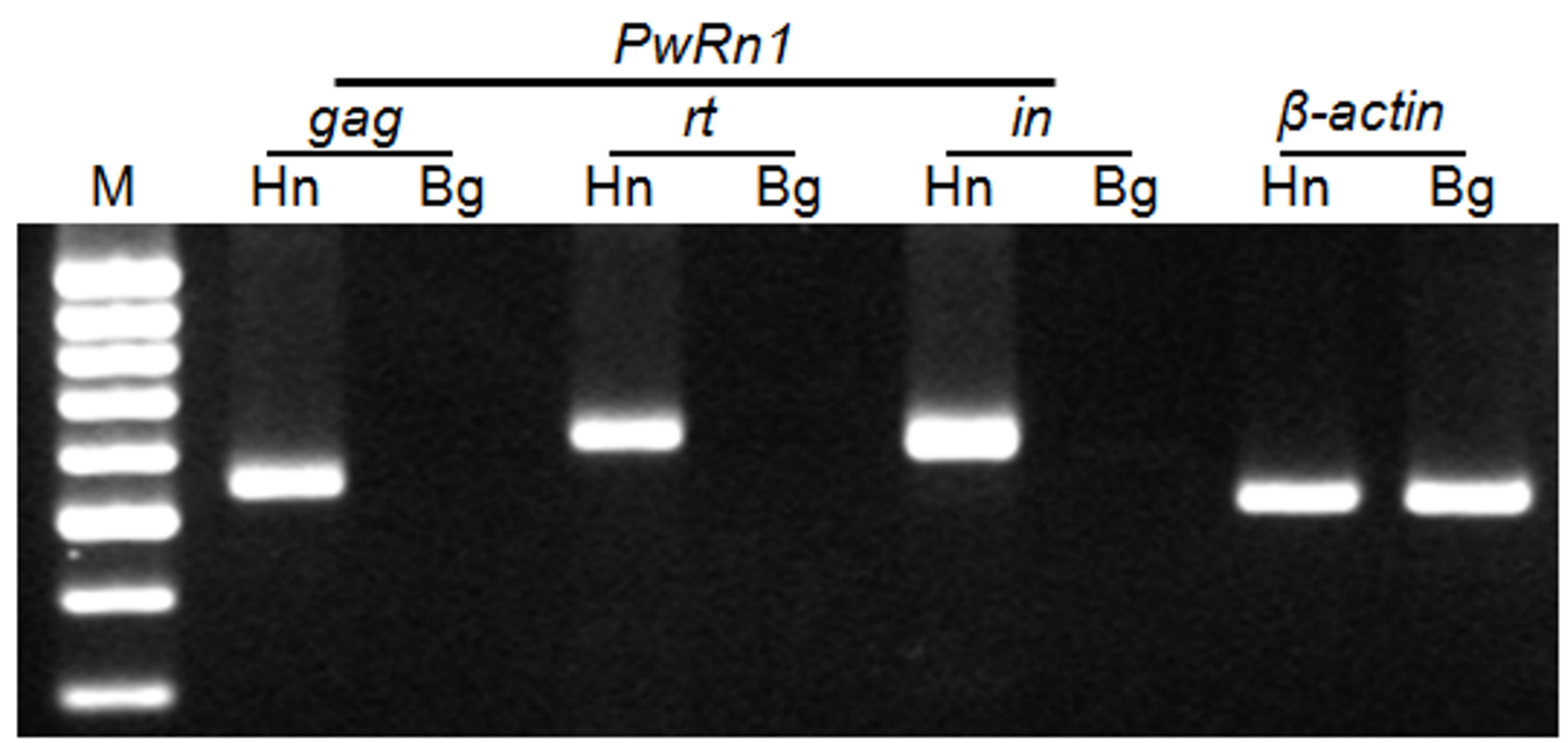

Figure 6

Amplification of PwRn I transcripts. Reverse-transcription PCR (RT-PCR) was performed with the total RNAs extracted from diploid (Haenam, $\mathrm{Hn}$ ) and triploid (Bogil-do, Bg) worms and PwRn I-specific primers. The RNAs were examined by conventional PCR to verify the absence of any contaminating genomic DNAs, prior to the RT-PCR (data not shown). The PCR products were analyzed by agarose gel-electrophoresis. A primer pair for the $\beta$-actin gene was used as a house-keeping gene control. M, I00-bp DNA ladder.

(VLP), which is thought to be an important intermediate in retrotransposition [25]. The origin and functional relevance of Gag found in the CsRn1-like elements could not be properly addressed, because neither cellular nor the other retrotransposon-related protein was detected during homolog searches based on the BLAST algorithm and Hidden Markov models. Considering the well-conserved, $\alpha$-helix-rich secondary structure (Additional file 2) and the active replication of these elements including CsRn1 [26], the proteins seem to have nucleic acid-binding capacity to form VLPs. Malik and Eickbush [7] have proposed the chimeric origin of LTR retrotransposons and retroviruses, between a preexisting element and non-LTR element/host gene. This may reflect the genetic flexibility endogenous in the mobile elements to generate novel functional/structural variants. A gag gene equipped in the preexisting element is likely to have been substituted by a presently unidentified host gene with the unusual motif during the evolutionary start point of CsRn1-like elements.

Different expression strategies were observed among the CsRn1-like elements (Figure 1 and 2; see also [15]). A single primary transcript is responsible for the production of multiple retrotransposon proteins, which are essential for the autonomous replication of the corresponding ele- ment, and is subjected to ribosomal frameshifting to maintain a proper ratio between the Gag and Pol proteins [27]. Genetic events including insertion/deletion of single nucleotide around the frameshifting region are occasionally attributable to the generation of retrotransposons with a single fused ORF. The Gag and Pol proteins are produced by proteolytic cleavage of a single translation product in the cases of single-ORF variants [28,29]. The variant form will replace the previous one in the host genome, via the highly efficient VLP formation [29]. The structural variation was not detected with the multiple PwRn1 copies retrieved by PCR. The ancient copies with two overlapped ORFs might have decayed in the Paragonimus genome, mainly due to the low propagation rate. Otherwise, diagnostic base substitutions in priming regions of the variant copies might lead to a failure in the amplification of the corresponding DNA segments.

Retrotransposons generally show very low retrotransposition and excision rates, and their expansion is largely restricted to germ cells and/or an early developmental stage [18]. These elements, however, become unstable under specific circumstances and can rapidly expand in the host genomes by increasing their replication frequency. The mobile potential of PwRn1 seemed to be differentially preserved among $P$. westermani populations; 
the element maintained its activity in diploids, while a majority of the activity had been suppressed in triploid individuals (Figures 5 and 6). In association with the origin of the triploid worms, an allopolyploidic hybridization occurred in ancestral diploids at the intra- or interspecies level has been suggested as a relevant genomic event ([13] and references therein). IRAP markers shared in the diploid and triploid worms are likely to represent genomic loci flanked by PwRn1 copies in the putative ancestral diploids, and may provide further genomic evidence supporting the proposed mechanism. Together with biotic/abiotic stresses such as aridity [30], polyploidization can boost the mobile potential of various retrotransposons [31,32]. Therefore, it was unexpected to find that $P w R n 1$ is less active in triploid genomes. Host genome surveillance for retrotransposons is likely to be reinforced in association with an increase in genomic dosage [33]. Alternatively, $P w R n 1$ itself would give a feedback effect as a mechanism to compensate for the amplified copy number, following the hybridization process. Phylogenetic and comparative analyses with the multiple PwRn1 copy sequences will be informative to elucidate the evolutionary status/mode of the element among the Paragonimus populations with different polyploidy.

There have been various reports on the comparison of $P$. westermani genomes to address the issue regarding the origin of polyploid genomes (reviewed in [13]). Molecular data such as the nucleotide sequences of mitochondrial and ribosomal DNAs dichotomized the genomes into the northeast (China, Japan, Korea and Taiwan) and south (the Philippines, Thailand and Malaysia) Asian groups, but failed in establishing more detailed relationships among the northeast populations with different polyploidy [34]. The retrointegration of retrotransposons is an irreversible event and the integration site is randomly selected $[35,36]$. These characteristics of mobile elements are particularly suitable for the comparative population studies at the intra- and interspecies levels $[37,38]$. In this study, we provided molecular evidence demonstrating genomic conservation and/or diversification between Korean diploids and triploids by analyzing intergenomic distributions of PwRn1 (Figure 5). The data suggests that IRAP and locus-specific typing of active retrotransposons can be an informative genetic marker in exploring the flow of haplotypes among $P$. westermani populations, and in elucidating the controversial hypotheses on the origin of the triploid populations. In plants, transposable elements are believed to be reactivated very early following polyploid formation and thus contribute to genetic diversity in the polyploidy species [41]. However, understanding on the role of these elements in polyploidy evolution is just beginning to emerge. The polyploid genomes are less common in animals than in plants. Our results on the suppressed mobile activity of PwRn1 in the triploid Paragonimus genomes can boost research to examine the evolutionary impacts of retrotransposons in polyploid animal genomes.

\section{Conclusion}

With the completion of genome projects from numerous eukaryotic organisms, comprehensive studies on the mobile genetic elements have been conducted with the whole genomic sequences. These studies have broadened our knowledge on the dynamics and impacts of retrotransposons in the host genomes. Despite the rapid accumulation of data, however, only limited information is currently available from a few species in the wide phylum Platyhelminthes. It is apparent that these invertebrate animal genomes contain diverse retrotransposons and that these elements are actively involved in the remodeling and diversification of their host genomes. Our results on the CsRn1-like elements of $P$. westermani and A. gambiae, especially in association with the diversified expression strategies, can make a significant step toward a better understanding of evolutionary episode within the unique CsRn1 clade of LTR retrotransposons, which is specifically expanded in the lower animal taxa. The differentially preserved mobile potential of $P w R n 1$ and resulting genomic polymorphism in diploid individuals will also be helpful in designing a comparative genomic study with the northeast Asian populations of $P$. westermani.

\section{Methods \\ Parasite}

Experimental dogs were orally challenged with the metacercariae of $P$. westermani, which were collected from naturally infected crayfish in endemic areas of Korea (Haenam, 2n; Bogil-do and Youngam, 3n) and China (Nanchang, Jiangxi Province, 2n) [39]. Five months after the infections, adult worms of the parasite were harvested from the dogs' lungs. The worms were washed five times with physiological saline at $4{ }^{\circ} \mathrm{C}$ and used for the extraction of DNA and RNA with the Wizard DNA Purification Kit (Promega, Madison, WI, USA) and TriZol reagents (Invitrogen, Carlsbad, CA, USA), according to the manufacturers' instructions. Materials from diploid worms (Haenam, Korea) were commonly used in this study; otherwise the sources were specifically indicated. The use of animals was approved by the Animal Ethics Committee of Korea Food and Drug Administration (protocol number NIH-05-09).

\section{Isolation of a CsRn I-like retrotransposon from $\mathbf{P}$. westermani}

The retrotransposon-related sequences were retrieved via a degenerate PCR method from the P. westermani genome, as described previously [14]. Of the sequences obtained, a clone (Pw-D-13), that showed a significant degree of 
sequence identity with CsRn1 was selected for further characterization. Genomic DNA library of the parasite constructed using the lambda FIX II vector system (Stratagene, La Jolla, CA. USA) was screened with the DNA probe according to the standard procedure of plaque-lift hybridization. The inserts of two positive clones were amplified by long-range PCR using primers designed from the vector regions (5'-CTAATACGACTCACTATAGGGCGTCG-3' and 5'-CCCTCACTAAAGGGAGTCGACTCG-3') and LA Taq polymerase, under the standard cycle condition (Takara, Shiga, Japan). The amplified products were digested with $K p n$ I and Xho I, and were cloned into pBluescript II SK(-) phagemid (Stratagene) for sequencing. The nucleotide sequences were automatically determined with the ABI PRISM 377 DNA Sequencer (Applied Biosystems, Foster City, CA, USA) and the BigDye Terminator Cycle Sequencing Reaction Kit (Perkin Elmer Corporation, Foster City, CA, USA). To ensure the accuracy of the reactions, nucleotide sequences from both strands were determined. Contig sequences were obtained by overlapping the sequence information. These contigs were then compared with one another to determine the full-unit of a retrotransposon encompassing the Pw-D-13 clone, which was designated P. westermani retrotransposon 1 (PwRn1).

\section{In silico identification of insect retrotransposons homologous to CsRn I}

Whole genomic sequences of A. gambiae and D. melanogaster deposited in the GenBank were surveyed by BLAST algorithms using the amino acid sequences of CsRn1 Gag protein, which showed a unique Cys motif [9], as a query. The hits showing amino acid positive values greater than $45 \%$ throughout the whole query sequence were subject to further analyses to determine the full-units of retrotransposons encompassing each of the gag genes. Multiple scaffold sequences were compared with one another and then, a common genetic element contained within them was isolated. The terminal repeats flanking a protein-encoding internal region were determined by analyzing the sequences with bl2seq at National Center for Biotechnology Information (NCBI, http:// www.ncbi.nlm.nih.gov/) and further verified by recognizing the duplicated target sequences from the direct upstream and downstream regions of the elements. BLAST searches with the entire nucleotide sequences of the elements were followed to confirm their integrity and to retrieve additional copy sequences from the identical databases. The coding profiles and conserved protein domains were predicted using ORF Finder at NCBI and InterProScan programs at European Bioinformatics Institute (EBI, http://www.ebi.ac.uk/Tools/InterProScan), respectively. The homology patterns were also determined by a series of BLAST searches against the non-redundant genomic/protein databases of the GenBank.

\section{Southern and dot-blot hybridization of PwRn I}

Genomic DNAs isolated from $P$. westermani adult worms (5 $\mu \mathrm{g}$ per each) were digested with restriction enzymes, Acs I, Sac I and Sfu I. After being separated on a $0.8 \%$ agarose gel, the restricted DNA fragments were transferred onto a nylon membrane (Hybond-N+; Amersham Pharmacia Biotech, Uppsala, Sweden) by capillary action in 10 $\times$ SSC. The blots were hybridized with the Pw-D-13 probe enzymatically labeled with the ECL Direct Labeling Kit (Amersham Pharmacia Biotech). The labeling, hybridizing, and signal detection conditions were determined under the manufacturer's instructions. For stringency washing, the membrane was washed twice in $0.1 \times$ SSC containing $6 \mathrm{M}$ urea and $0.4 \% \mathrm{SDS}$ at $42^{\circ} \mathrm{C}$ for $20 \mathrm{~min}$, and twice in $2 \times \mathrm{SSC}$ at room temperature for $5 \mathrm{~min}$.

Various amounts of the Pw-D-13 DNA fragment were blotted onto a nylon membrane, according to the standard procedure of dot-blot hybridization. The membrane was hybridized with the genomic probe of $P$. westermani, which was prepared by sonicating the genomic DNA into small DNA fragments between 0.5 and $2 \mathrm{~kb}$. For a singlecopy control, a portion of the Paragonimus cysteine protease gene [GenBank:U70537], with a size of approximately $700 \mathrm{bp}$, was amplified from the parasite genome by PCR using a gene-specific primer pair (5'-TCAGTTGTCTTGTTGTCGTGG-3' and 5'-TGCCTGTTTCTCCTCATTCTTG-3') and blotted onto the membrane. Probe labeling and hybridizing conditions were identical to those for Southern blot hybridization. The intensities of signals were measured using the LAS-1000plus system (FUJIFILM, Tokyo, Japan) and compared with those of the control gene.

\section{Reverse-transcription PCR (RT-PCR)}

Total RNAs were extracted from the diploid and triploid adult worms (10 worms per each) and treated with the RNase-free DNase (GIBCO BRL, Rockvile, MD, USA). Reverse transcription and following amplification of $P w R n 1$ transcripts were carried out with the total RNAs and retrotransposon-specific primers, using RNA PCR Kit (AMV) (Ver. 2.1; Takara) under the manufacturer's instructions. The primers used were as follows: 5'-AGAGAGATGTGGCTACAGCG-3' and 5'-GTTTAAGTCGTGGACCTCAG-3' for gag; 5'GCAGGTTGACACCAGACAAG-3' and 5'-ATCGGTTAACGGTCGGATAC-3' for $r t$; 5'-GTCGATGCAATCCGTTGGAC-3' and 5'-CCAGTGCACCCTACGACCTG-3' for in; 5'-GGCCATGTACGTTGCTATCC-3' and 5'-CAGAGAGAACAGTGTTGGCG-3' for $\beta$-actin gene. The absence of any contaminating DNA was confirmed by preparing reactions without reverse transcriptase during the first round of cDNA synthesis and the $\beta$-actin gene was selected for a house-keeping gene control. The reaction products were resolved by agarose gel electrophoresis. 


\section{Inter-retrotransposon amplified polymorphism (IRAP)}

Primers were designed to match the 5'- and 3'-ends of a consensus PwRn1 LTR sequence in the outward directions, respectively (LTR-5R, 5'-AGGCAGGCCAGTGAAATCT-3' and LTR-3F, 5'CGACTTAGTGCAACGAGCAC-3'). Genomic DNAs were individually extracted from diploid and triploid worms. The IRAP PCR was performed with reaction mixtures containing $20 \mathrm{ng}$ of the genomic DNA, $1 \mu \mathrm{M}$ of each primer, $0.2 \mathrm{mM}$ of each dNTP precursors, $1.6 \mathrm{mM}$ of $\mathrm{MgCl} 2$, and 1.25 units of Taq polymerase (Takara) in $2 \mathrm{mM}$ Tris- $\mathrm{HCl}$ buffer ( $\mathrm{pH}$ 8.0). PCR cycling parameters were as follows: $4 \mathrm{~min}$ at $94^{\circ} \mathrm{C} ; 35$ cycles of $40 \mathrm{sec}$ at $96^{\circ} \mathrm{C}, 40 \mathrm{sec}$ at $56^{\circ} \mathrm{C}$, and $2 \mathrm{~min}$ at $72^{\circ} \mathrm{C} ; 10 \mathrm{~min}$ at $72^{\circ} \mathrm{C}$. The PCR products were analyzed by electrophoresis on 2\% agarose gels (NuSieve 3:1 agarose; Cambrex Bio Science, Rockland, ME, USA) and visualized by staining with ethidium bromide.

\section{Phylogenetic analysis}

Pol protein sequences of LTR retrotransposons were aligned with ClustalX and optimized with GeneDoc programs. The regions corresponding to each of the RT, RH and IN domain sequences were selected from the alignment, in order to increase the analytical resolution, compared to that obtained by using RT sequences alone $[22,40]$. The resulting concatenates comprising approximately 510 amino acid positions were adopted in a phylogenetic analysis for the construction of maximum likelihood tree using the quartet method implemented in TREE-PUZZLE (Ver. 5.2). The analytical options were as follows: JTT model for substitution, estimation of invariant site proportion from the input data, 1,000 puzzling steps assuming rate heterogeneity with eight gamma categories (the gamma distribution parameter alpha was estimated from the data set). Indels between pairs of sequences were regarded as missing data. Tree construction was performed by the neighbor-joining method. Gag sequences of the CsRn1-clade members were similarly examined by the phylogenetic approach. The phylogenetic trees were displayed by the TreeView program.

\section{Abbreviations}

ENV: envelope; IN: integrase; IRAP: inter-retrotransposon amplified polymorphism; LTR: long terminal repeat; ORF: open reading frame; PR: protease; $\mathrm{RH}$ : RNase H; RT: reverse transcriptase; TSD: target site duplication; VLP: virus-like particle.

\section{Authors' contributions}

YAB contributed to the experimental design, sequence analyses, sequence alignments and phylogenetic analyses, and drafted the manuscript. JSA performed experiments regarding construction and screening of the P. westermani genomic DNA library. SHK carried out the Southern and dot-blot hybridizations, and IRAP analysis. MGR participated in experimental design and bioinformatic analysis of the sequence data. YK helped to collect the experimental materials and to design the project. SYC oversaw the research project, contributed in its design and participated in editing the manuscript. All authors read and approved the final manuscript.

\section{Additional material}

\section{Additional File 1}

Primer-binding site (PBS) and polypurine tract (PPT) conserved in the nucleotide sequences of PwRn1 and AgCRs. Nucleotides in both termini of LTRs, PBS and PPT were compared among these CsRn1-like elements. The 3'-end of bovine tRNATrp, complement to the putative PBS, is also presented at the top. Dots were introduced into the sequences to increase their homology values. Breaks marked with a double slash indicate the regions which were removed to shorten the alignment. Click here for file

[http://www.biomedcentral.com/content/supplementary/14712164-9-482-S1.tiff]

\section{Additional File 2}

Comparison of Gag sequences encoded by the CsRn1-clade members. The amino acid sequences were aligned with ClustalX and optimized with GeneDoc. The shading pattern indicates difference in amino acid conservation for the individual positions and sequence identities are highlighted in black. The functional signature (CHCC) conserved in the CsRn1-like retrotransposons is marked with filled arrowheads. The filled cylinders at the top indicate regions corresponding to the $\alpha$-helixes.

Click here for file

[http://www.biomedcentral.com/content/supplementary/14712164-9-482-S2.tiff]

\section{Acknowledgements}

This work was supported by a grant from the Korea Science and Engineering Foundation (KOSEF; 1999 2-208-004 5) to SYC and partly by a National Research and Development Program of the National Institute of Health (the Anti-Communicable Diseases Control Program, NIH 348-6I I I-2I5) to $\mathrm{YAB}$.

\section{References}

I. Boeke JD, Garfinkel DJ, Styles CA, Fink GR: Ty elements transpose through an RNA intermediate. Cell I985, 40:49I-500.

2. Jurka J, Kapitonov VV: Sectorial mutagenesis by transposable elements. Genetica 1999, 107:239-248.

3. Lim JK, Simmons MJ: Gross chromosome rearrangements mediated by transposable elements in Drosophila melanogaster. Bioessays 1994, 16:269-275.

4. Hudakova S, Michalek W, Presting GG, ten Hoopen R, dos Santos K, jasencakova Z, Schubert I: Sequence organization of barley centromeres. Nucleic Acids Res 200I, 29:5029-5035.

5. McDonald JF: Macroevolution and retroviral elements. Bioscience 1990, 40:183-191.

6. Kidwell MG, Lisch D: Transposable elements as sources of variation in animals and plants. Proc Natl Acad Sci USA 1997, 94:7704-77II.

7. Malik HS, Eickbush TH: Phylogenetic analysis of ribonuclease $\mathbf{H}$ domains suggests a late, chimeric origin of LTR retrotransposable elements and retroviruses. Genome Res 200I, II:II87-II 97. 
8. Lloréns C, Futami R, Bezemer D, Moya A: The Gypsy database (GyDB) of mobile genetic elements. Nucleic Acids Res 2008, 36:D38-D46.

9. Bae YA, Moon SY, Kong Y, Cho SY, Rhyu MG: CsRnI, a novel active retrotransposon in a parasitic trematode, Clonorchis sinensis, discloses a new phylogenetic clade of Ty3/gypsy-like LTR retrotransposons. Mol Biol Evol 200।, I 8: | 474- | 483.

10. Dalle Nogare DE, Clark MS, Elgar G, Frame IG, Poulter RT: Xena, a full-length basal retroelement from tetraodontid fish. Mol Biol Evol 2002, 19:247-255.

II. Butler M, Goodwin T, Poulter R: An unusual vertebrate LTR retrotransposon from the cod Gadus morhua. Mol Biol Evol 200I, I 8:443-447.

12. DeMarco R, Kowaltowski AT, Machado AA, Soares MB, Gargioni C, Kawano T, Rodrigues V, Madeira AM, Wilson RA, Menck CF, Setubal JC, Dias-Neto E, Leite LC, Verjovski-Almeida S: Saci-I, -2, and -3 and Perere, four novel retrotransposons with high transcriptional activities from the human parasite Schistosoma mansoni. J Virol 2004, 78:2967-2978.

13. Blair D, Xu ZB, Agatsuma T: Paragonimiasis and the genus Paragonimus. Adv Parasitol 1999, 42: I I3-222.

14. Bae YA, Kong Y: Divergent long-terminal-repeat retrotransposon families in the genome of Paragonimus westermani. Korean J Parasitol 2003, 41:221-23I.

I5. Tubío JM, Naveira H, Costas J: Structural and evolutionary analyses of the Ty3/gypsy group of LTR retrotransposons in the genome of Anopheles gambiae. Mol Biol Evol 2005, 22:29-39.

16. Copeland CS, Brindley PJ, Heyers O, Michael SF, Johnston DA, Williams DL, Ivens AC, Kalinna BH: Boudicca, a retrovirus-like long terminal repeat retrotransposon from the genome of the human blood fluke Schistosoma mansoni. J Virol 2003, 77:6153-6166.

17. Holt RA, Subramanian GM, Halpern A, Sutton GG, Charlab R, Nusskem DR, Wincker P, Clark AG, Ribeiro JM, Wides R, et al.: The genome sequence of the malaria mosquito Anopheles gambiae. Science 2002, 298: 129-149.

18. Boeke JD, Stoye JP: Retrotransposons, endogenous retroviruses, and the evolution of retroelements. Edited by: Coffin JM, Hughes SH, Varmus HE. Cold Spring Harbor Laboratory press; 1997.

19. Jordan IK, McDonald JF: Tempo and mode of Ty element evolution in Saccharomyces cerevisiae. Genetics 1999, I 5 I:134 I-I35 I.

20. Eickbush TH: Origin and evolutionary relationships of retroelements. Edited by: Morse SS. Raven press; 1994.

21. Engelman A, Hickman AB, Craigie R: The core and carboxyl-terminal domains of the integrase protein of human immunodeficiency virus type I each contribute to nonspecific DNA binding. J Virol 1994, 68:5911-5917.

22. Malik HS, Eickbush TH: Modular evolution of the integrase domain in the Ty3/Gypsy class of LTR retrotransposons. J Virol 1999, 73:5186-5190.

23. Jurka J: Evolutionary impact of human Alu repetitive elements. Curr Opin Genet Dev 2004, I 4:603-608.

24. Abe H, Ohbayashi F, Shimada T, Sugasaki T, Kawai S, Mita K, Oshiki $\mathrm{T}$ : Molecular structure of a novel gypsy -Ty3-like retrotransposon (Kabuki) and nested retrotransposable elements on the W chromosome of the silkworm Bombyx mori. Mol Gen Genet 2000, 263:916-924.

25. Roth JF: The yeast Ty virus-like particles. Yeast 2000, 16:785-95

26. Bae YA, Kong Y: Evolutionary courses of CsRn I long-terminalrepeat retrotransposon and its heterogeneous integrations into the genome of the liver fluke, Clonorchis sinensis. Korean J Parasitol 2003, 41:209-2 I9.

27. Dinman JD, Wickner RB: Ribosomal frameshifting efficiency and gag/gag-pol ratio are critical for yeast $M I$ doublestranded RNA virus propagation. J Virol 1992, 66:3669-3676.

28. Levin HL, Weaver DC, Boeke JD: Novel gene expression mechanism in a fission yeast retroelement: Tfl proteins are derived from a single primary translation product. $E M B O \mathrm{~J}$ 1993, I 2:4885-4895.

29. Kalmykova Al, Kwon DA, Rozovsky YM, Hueber N, Capy P, Maisonhaute C, Grozdev VA: Selective expansion of the newly evolved genomic variants of retrotransposon I73I in the Drosophila genomes. Mol Biol Evol 2004, 2 I:228I-2289.

30. Kalendar R, Tanskanen J, Immonen S, Nevo E, Schulman AH: Genome evolution of wild barley (Hordeum spontaneum) by $B A R E-I$ retrotransposon dynamics in response to sharp microclimatic divergence. Proc Natl Acad Sci USA 2000 , 97:6603-6607.

31. Evgen'ev MB, Zelentsova H, Shostak N, Kozitsina M, Barskyi V, Lankenau DH, Corces VG: Penelope, a new family of transposable elements and its possible role in hybrid dysgenesis in Drosophila virilis. Proc Natl Acad Sci USA 1997, 94: I96-20I.

32. Labrador M, Farre M, Utzet F, Fontdevila A: Interspecific hybridization increase transposition rates of Osvaldo. Mol Biol Evol 1999, 16:931-937.

33. Cam HP, Noma K, Ebina H, Levin HL, Grewal SI: Host genome surveillance for retrotransposons by transposon-derived proteins. Nature 2008, $451: 431-436$

34. Iwagami M, Ho LY, Su K, Lai PF, Fukushima M, Nakano M, Blair D, Kawashima K, Agatsuma T: Molecular phylogeographic studies on Paragonimus westermani in Asia. I Helminthol 2000, 74:315-322.

35. Okada N: SINEs. Curr Opin Genet Dev 1991, I:498-504.

36. Kido $Y$, Saitoh M, Murata S, Okada N: Evolution of the active sequences of the $\mathrm{Hpa} I$ short interspersed elements. J Mol Evol 1995, 41:986-995.

37. Takahashi K, Terai $Y$, Nishida M, Okada N: A novel family of short interspersed repetitive elements (SINEs) from cichlids: the patterns of insertion of SINEs at orthologous loci support the proposed monophyly of four major groups of cichlid fishes in lake Tanganyika. Mol Biol Evol 1998, I 5:391-407.

38. Nikaido M, Rooney AP, Okada N: Phylogenetic relationships among cetartiodactyls based on insertions of short and long interspersed elements: hippopotamuses are the closest extant relatives of whales. Proc Natl Acad Sci USA 1999, 96:10261-10266.

39. Park GM, Lee KJ, Im KI, Park H, Yong TS: Occurrence of a diploid type and a new first intermediate host of a human lung fluke, Paragonimus westermani, in Korea. Exp Parasitol 2001, 99:206-212

40. Xiong $\mathrm{Y}$, Eickbush TH: Origin and evolution of retroelements based upon their reverse transcriptase sequences. EMBO 1990, 9:3353-3362.

4I. Comai L, Tyagi AP, Winter K, Holmes-Davis R, Reynolds SH, Stevens $Y$, Byers $B$ : Phenotypic instability and rapid gene silencing in newly formed Arabidopsis allotetraploids. Plant Cell 2000, I2:155 I-1568.
Publish with Biomed Central and every scientist can read your work free of charge

"BioMed Central will be the most significant development for disseminating the results of biomedical research in our lifetime. "

Sir Paul Nurse, Cancer Research UK

Your research papers will be:

- available free of charge to the entire biomedical community

- peer reviewed and published immediately upon acceptance

- cited in PubMed and archived on PubMed Central

- yours - you keep the copyright

Submit your manuscript here:

http://www.biomedcentral.com/info/publishing_adv.asp 